\title{
Breast Cancer in Young Women - News from the BCY3 Consensus Conference
}

\author{
Chair: \\ Shani Paluch-Shimon ${ }^{a}$ \\ Participants: Sibylle Loibl ${ }^{b}$ Fedro A. Peccatori ${ }^{c}$ Elżbieta Senkus-Konefka ${ }^{d}$ \\ a Breast Cancer Service for Young Women, Fellow of the Dr Pinchas Borenstein Talpiot Medical Leadership Program, Oncology Institute, \\ Tel Hashomer, Israel; \\ ${ }^{b}$ GBG Forschungs GmbH, Neu-Isenburg, Germany; \\ ${ }^{c}$ Fertility \& Procreation Unit, Division of Gynecologic Oncology, European Institute of Oncology, Milan, Italy; \\ ${ }^{\mathrm{d}}$ Klinika Onkologii i Radioterapii, Uniwersyteckie Centrum Kliniczne, Gdańsk, Poland
}

Breast cancer in women under 40 represents $6-7 \%$ of all new breast cancer diagnoses. Breast cancer in young women is particularly challenging as there are no established screening tools for early detection, thus, the disease is often diagnosed at a more advanced stage and with less favorable biology than in older women, and there is a multitude of psychosocial issues that are unique to young women. Additionally, there is only limited research on breast cancer in young women. In 2012 the European School of Oncology (ESO) hosted the first dedicated conference on breast cancer in young women (BCY1) and published the first international consensus guidelines on breast cancer in young women. There have since been 2 further conferences (followed by guideline updates) - the most recent, BCY3, in Lugano in November 2016 - sponsored by both ESO and ESMO. Members of the BCY3 faculty generously agreed to participate in this expert panel to discuss key issues and questions pertaining to breast cancer in young women. In this discussion experts from 3 different countries share their opinions.

Shani Paluch-Shimon, Tel Hashomer

\section{Question 1: Do You Think there Is Convincing Evidence that the Biology of Tumors Is Different in Young Women and If Yes, How Should this Impact Our Clinical Practice?}

Loibl: This has often been discussed as a reason of the inferior prognosis of young women with breast cancer, especially those with a hormone receptor positive $(\mathrm{HR}+)$ tumor. In young women, triple negative breast cancer (TNBC) in diagnosed in up to $35 \%$ of the patients. The incidence of germline BRCA (gBRCA) mutations in women younger than 40 years is higher than in older women especially if the young women are diagnosed with TNBC. In a paper by Azim et al. the inferior outcome especially in young women with $\mathrm{HR}+$ primary breast cancer indicates that age is the only dismal prognostic factor in this cohort, whereas in triple negative and HER2+ breast cancer young women do not have a worse prognosis compared to other age cohorts. An investigation by Anders et al. looked at the gene expression data in young women compared to older women and found different gene expression patterns, suggesting a different biology of young breast cancer patients. Although I am convinced that the biology in young breast cancer patients is different, to date we have no data supporting that young women should be treated differently from other breast cancer patients. With the exception that we need to discuss more often an endocrine therapy consisting of an LHRH analogue plus an aromatase inhibitor, based on the data by the SOFT trial, showing the greatest DFS benefit by this treatment in women younger than 35 years. We also will need to offer/perform more often genetic counselling as well as fertility counselling.

Peccatori: Young women have a higher incidence of triple negative, luminal B and HER2+ breast cancers compared to older women. Nonetheless, within each subgroup the intrinsic difference between younger and older women is not so clear. Recent evidence shows interesting hints that indicate specific biological differences. Some are tumor-related, with different gene expression profiles or genetic abnormalities in tumors occurring in young women (e.g. GATA3 somatic mutations), others are stroma-related, including infiltrating immune cells and their function. Our group has recently published data on a selected population of young women diagnosed with breast cancer during pregnancy. We reported a similar immuno-histochemical profile for estrogen receptor (ER),

\section{KARGER}

(๑) 2016 S. Karger GmbH, Freiburg
Shani Paluch-Shimon, MBBS, MSc Breast Cancer Service for Young Women Oncology Institute, Sheba Medical Center Tel Hashomer 52621, Israel Shani.Paluch-Shimon@sheba.health.gov.il 
progesterone receptor (PgR) and HER2 compared to age-matched non-pregnant controls, and comparable mutation rate of PIK3CA and p53. Nevertheless, a lower incidence of lymphocyte rich tumors was reported, with higher expression of RANK-L and mammary stem cell-specific genes. In the general population of young women with breast cancer, a higher incidence of high penetrance inherited genetic abnormalities, such as BRCA1 and BRCA2 mutations, has been reported, with different incidence in different populations. As there are epidemiological data suggesting a worse prognosis for luminal tumors, it is possible that endocrine resistance might be commoner than thought. Commercially available gene expression profiles have been validated also in young women and might be useful in identifying patients with luminal tumors that can avoid chemotherapy.

Senkus-Konefka: Yes, I think so. Data on which there is convincing evidence include, among others, different distribution of intrinsic phenotypes, higher mortality within the phenotype (in particular for luminal A) and higher local recurrence risk. How should this impact clinical practice? My impression is that a major field of improvement for luminal tumors is the improvement of endocrine treatment (ET) - in particular, prior to the SOFT-TEXT data ovarian suppression was used in a rather chaotic way and we are still not sure about the optimal endocrine therapy in this population. Again, this is my personal opinion, but in luminal tumors probably the very young patients are much more dependent on the ER stimulation (or rather it's more difficult to obtain adequate suppression of ER pathway because of difficulties in obtaining ovarian suppression and possibly inadequate ER blockade in view of a generally higher ovarian reserve), so possibly more 'aggressive' ET options (i.e. more ovarian function suppression) should be used, even if patients are not given chemotherapy (as it is recommended, based on SOFT conclusions).

Particular attention must be paid to ensuring adequate ovarian suppression in patients on aromatase inhibitors (AI), and estrogen/ FSH levels should be checked on a regular basis, since use of AI in case of incomplete ovarian suppression results in ovarian stimulation and may lead to increased estrogen concentrations. The decision on the use of AI in premenopausal patients should also take into account contradictory (albeit quite immature) data on the impact of their use on overall survival (suggested increase in the risk of death in the ABCSG study). In TNBC and HER2+ the clinical practice should not differ from that in other age groups. Regarding loco-regional management, surgery should follow the rules used in other age groups; radiotherapy (RT) should take into account the increased risk of loco-regional failures, which should be mostly reflected by (i) including age as one of the risk factors (but not the only risk factor) in decisions on postmastectomy RT; (ii) obligatory use of tumor bed boost in patients receiving breast conserving therapy (BCT). Additionally, due to expected long survival and the risk of serious late sequelae, such as contralateral breast cancer and cardiac toxicity, particular attention must be paid to optimal treatment technique.

\section{Question 2: What Do You Think Are the Biggest Obstacles in Women with Breast Cancer Accessing Fertility Preservation?}

Loibl: Many young and very young women with breast cancer are overwhelmed by the diagnosis and do not want to discuss fertility issues at the same time as discussing the best therapy. On the one hand we need to discuss the possible gonadotoxic therapies with the patients and the possible interventions to protect fertility; on the other hand some of these interventions need time and might delay commencement of treatment, especially systemic therapy. This is especially problematic when neoadjuvant chemotherapy is being discussed.

Peccatori: As young breast cancer patients are frequently treated with chemotherapy that might reduce subsequent fertility, oncofertility counseling should be offered to all young women with breast cancer, just after the diagnosis. Unfortunately, still few specialized breast units offer this kind of service and many patients are left with the regret that fertility issues have not been discussed. In many countries, the access to fertility preservation through oocyte harvesting is an added direct cost, and not all fertility centers feel comfortable in offering such procedures. This is of course a big obstacle to access to fertility preservation. Moreover, even if there have been extensive publications about ovarian stimulation protocols in breast cancer, the optimal regimen, the optimal number of oocyte to cryopreserve, the possible integration among different techniques including pharmacological ovarian protection and ovarian cryopreservation is not yet clear. Thus, the implementation of an oncofertility network with shared goals and research objectives is essential.

Senkus-Konefka: This is probably very country- and culture-related, but possibly the biggest obstacle is in the mentality of physicians (who are worried about the aggressive course of disease in young patients and want to start treatment as soon as possible) and patients (who under the trauma of being diagnosed with breast cancer are overwhelmed with information and petrified with anxiety, and often place fertility issues lower on their list of priorities). Possible issues would also be inadequate education of physicians about the technical possibilities of fertility preservation and about the safety of ovarian stimulation necessary for banking embryos or oocytes (both in terms of treatment delay and the exposure to hormonal manipulations). Another obstacle might be problems with financing fertility preserving techniques (not reimbursed at least in some countries and being beyond the financial abilities of many (most?) young women). Patients in some countries also face legal restrictions, in particular when technologies involving freezing embryos are used. Another problem may be a lack of local expertise in using techniques other than freezing embryos. 


\section{Question 3: In Healthy Young Women Not at an Increased Risk of Breast Cancer, Is There a Rationale and Justification for Clinical Trials Evaluating Screening for Early Detection?}

Loibl: If a woman is not at an increased risk, there is probably no justification to even conduct a clinical trial investigating the role of screening measures. Screening procedures in some countries include women from the age of 40 . However, the net benefit in this younger age group is even more questionable than in women from the age of 50 onwards, when the incidence starts to rise. In general, I would not support such a clinical trial, because the incidence without additional risk factors is low. This trial would be huge and from logistical and other reasons impossible to be conducted in a timely fashion.

Peccatori: Breast cancer is relatively rare in women below the age of 40 , representing only $5 \%$ of all breast cancers. Nonetheless, the absolute numbers are not trivial and in some low-middle income countries the proportion of cancer in young women may be as high as $25 \%$. Breast cancer screening is an effective and life-saving procedure when applied to age-specific cohorts, where screening mammography (or more recently tomosynthesis) has demonstrated a clear reduction in breast cancer mortality. In women less than 40 years of age, not at an increased risk of breast cancer, at present there is no justification for breast cancer screening. Increased breast density typical of young age and the need of intravenous gadolinium for MRI, hinder the feasibility of a population based radiological screening. Future possibilities of risk-adapted screening or more sensitive and specific 'liquid screening' trough blood or urine samples will possibly re-open the issue in the near future.

Senkus-Konefka: In view of the ongoing discussion on the role of mammography even in women $>50$ and the data on minor benefit of mammography screening in younger populations, I don't think there is any place for screening mammography in women $<50$. The development of other imaging technologies (such as $3 \mathrm{D}$ ultrasound) may create a room for testing this modality in younger patients - also taking into account higher sensitivity of ultrasound in young women. I general, however, I don't think any imaging may be sufficiently cost-effective to legitimize its use in mass screening of women $<50$ (in view of the expected low yield of cancers). The future could be any kind of blood/urine test assessing e.g. circulating tumor DNA or a metabolomics profile, provided the cost of the test in a low-incidence population would justify its clinical use.

\section{Question 4: What Should Be the Key Research Priorities for Breast Cancer in Young Women?}

Loibl: This is an important question. First of all, young breast cancer patients should not be excluded from clinical trials, espe- cially when a HR+ breast cancer has been diagnosed. The way CDK4/6 inhibitors are currently developed is a step in the right direction. One probably very difficult question to solve would be to investigate the role of non-gBRCA mutation carriers. These women are young and insecure because a mutation has been detected (e.g., PALB2) but the same treatment is offered as in nonBRCA carriers, because we have no data. This is a difficult problem, because these mutations are very rare, but I think an effort to collect data on current practice and outcome could help.

Another focus of research could be the role of radiotherapy. The trials to my knowledge have all been conducted in postmenopausal low-risk patients, but there are also premenopausal low-risk patients. In general we need to carefully address the question which very young woman might be spared more intensive therapy. Many questions regarding quality of life and optimal therapy regarding the wish to become a mother amongst other questions indirectly related to therapy have not well been addressed in young breast cancer patients.

Peccatori: Biology, genetics, and psychological adaptation to the trauma of diagnosis and treatment are to me the key research priorities for breast cancer in young women. We are still missing an international age and population specific tissue bank to investigate the biological peculiarities of breast cancer in young women. Even for the quite frequent $B R C A 1$ and $B R C A 2$ mutations we do not know yet what is the best onco-preventative strategy and at what age it should be implemented. Specific mutations or single nucleotide polymorphisms (SNPs) may influence the probability of contralateral breast or ovarian cancer and should be investigated within a clear data sharing policy. Trials with platinum compounds and PARP inhibitors are ongoing and will hopefully improve prognosis as in ovarian cancer affected carriers. Young women with breast cancer are not special, but they are different. Specific psychosocial needs should be individually investigated and addressed, including fertility preservation and breast cancer during pregnancy. Adherence and non-interruption of effective endocrine treatment should be implemented through better communication and with a more proactive management of side effects.

Senkus-Konefka: What I feel we are missing most, is more data on the very young patients $(<35)$ in terms of specificities of their tumor biology, genetic predispositions beyond BRCA1/2 (with a potential for targeting these genetic abnormalities) or role of multigene expression testing. I also share the feeling that premenopausal patients are insufficiently included in endocrine therapy trials and it should be a priority of the whole breast cancer society to insist on stopping dividing patients' inclusion into clinical trials according to their 'formal' menopausal status as long as they undergo effective ovarian suppression.

A huge unmet need is of course the TNBC, which affects young women more often than other populations. This leaves space for post-neoadjuvant studies, testing new treatments in selected populations of patients with particularly dismal prognosis. Metastatic 
TNBC creates possibilities of for developing new agents and strategies for particular biological subtypes of TNBC. For both these tasks a broad international collaboration is necessary, taking into account the rarity of particular conditions.

The other possible subject would be treatment de-escalation (in particular of drugs impacting the fertility). Here probably the most space exists in HER2-positive and luminal tumors, where optimal (possibly combined) targeted therapy (including the antibody-drug conjugate T-DM1) can potentially replace classical cytotoxic chemotherapy, at least in some patients.

\section{Participants}

Prof. Dr. med. Sibylle Loibl

Chair of the German Breast Group

GBG Forschungs GmbH

Martin Behaim Strasse 12

63263 Neu-Isenburg, Germany

Sibylle.Loibl@GBG.de

Fedro Alessandro Peccatori, MD PhD

Fertility \& Procreation Unit

Division of Gynecologic Oncology

European Institute of Oncology

Via Ripamonti 435

20141 Milan, Italy

fedro.peccatori@ieo.it

Dr. med. Elżbieta Senkus-Konefka

Klinika Onkologii i Radioterapii

Uniwersyteckie Centrum Kliniczne

ul. Dębinki 7

80-952 Gdańsk, Poland

elsenkus@gumed.edu.pl 


\section{Erratum}

Breast Care

DOI: $10.1159 / 000475565$

In the expert discussion

Breast Cancer in Young Women - News from the BCY3 Consensus

Conference (Breast Care 2016;11:432-435; DOI 10.1159/000454873), the address of Dr. Senkus-Konefka was incorrect.

The correct affiliation is:

Elżbieta Senkus-Konefka, MD, PhD

Department of Oncology \& Radiotherapy

Medical University of Gdańsk

Dębinki 7, 80-211 Gdańsk, Poland

elsenkus@gumed.edu.pl 